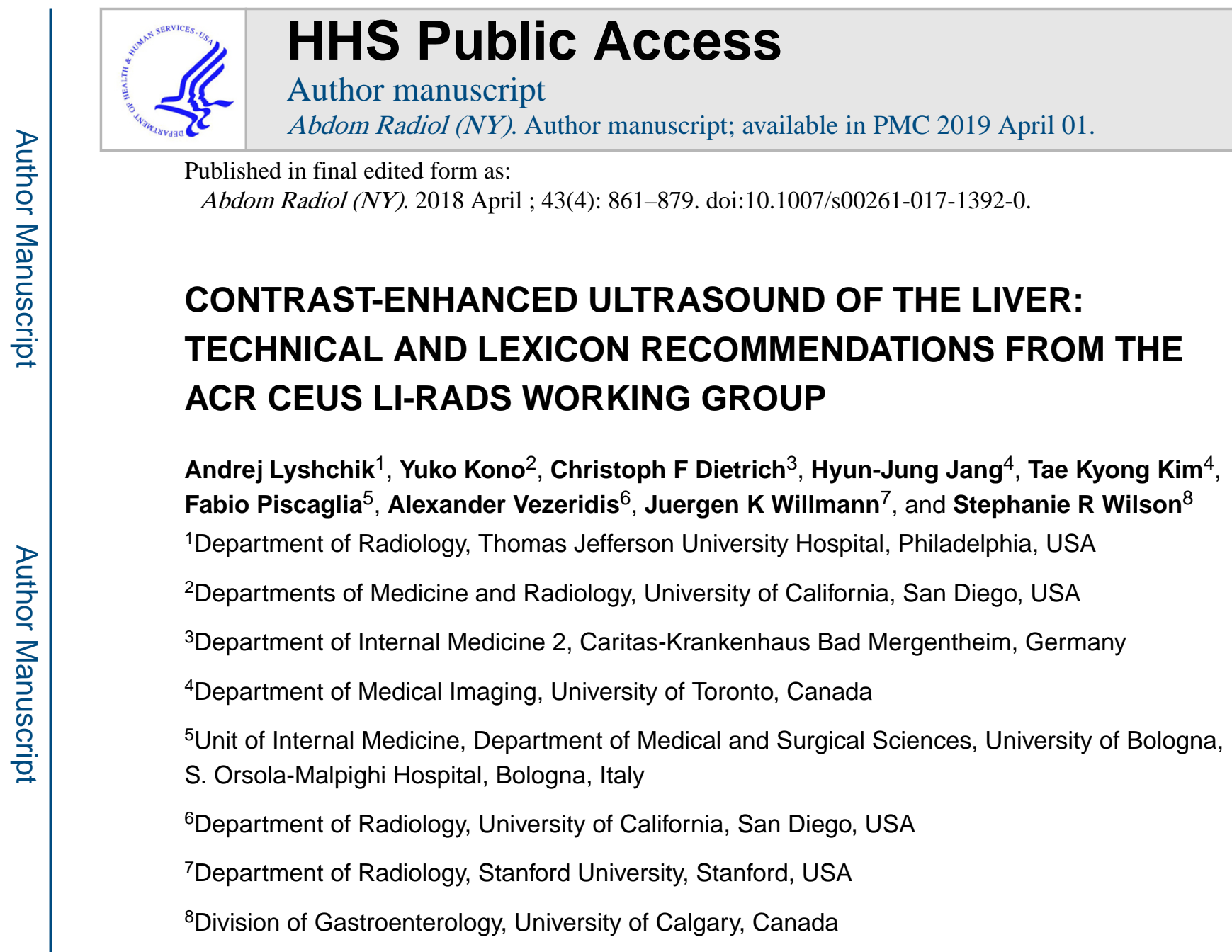

\title{
Introduction
}

Hepatocellular carcinoma (HCC) is the fifth most common cancer worldwide with an annual incidence of over 750,000, predominantly affecting patients with cirrhosis and chronic hepatitis (1). Treatment options for HCC include surgery, local ablation, embolization, and hepatic transplantation which are more feasible and effective when performed in the early tumor stage. Therefore, early detection and accurate diagnosis of HCC have become increasingly important (2). Current American Association for the Study of Liver Diseases (AASLD) guidelines for the treatment of hepatocellular carcinoma recommend ultrasound

Corresponding Author: Andrej Lyshchik, MD, PhD, Associate Professor of Radiology, Thomas Jefferson University Hospital, $132 \mathrm{~S}$. 10th Street, 763G Main Bldg, Philadelphia, PA 19107, Office: 215-503-0587, FAX: 215-955-8549, Andrej.Lyshchik@ jefferson.edu.

Compliance with Ethical Standards:

This article does not contain any studies with human participants or animals performed by any of the authors.

Conflict of Interest:

Andrej Lyshchik: Research support: GE, Siemens, Toshiba; Advisory Board: Bracco; Speaker: GE, SonoScape

Yuko Kono: Research support: Toshiba, GE, Lantheus

Christoph F Dietrich: Advisory Board; Hitachi, Mindray, JAZZ. Speaker: Hitachi, Supersonic, Siemens, Mindray, GE, Bracco, Pentax,

Novartis.

Hyun-Jung Jang: none

Tae Kyong Kim: none

Fabio Piscaglia: Research support: Esaote; Advisory board: Bayer, Eisai; Speaker: Bracco, Bayer

Alexander Vezeridis: none

Juergen K Willmann: Research support: Siemens, GE, Philips, Bracco; Consulting: Bracco, Triple Ring Technologies; Advisory

Board: Lantheus, Bracco, SonoVol

Stephanie R Wilson: Research support: Siemens, Philips and Samsung; Speaker: GE and Samsung 
(US) for HCC surveillance, and computed tomography (CT) and magnetic resonance imaging (MRI) for diagnosis of $\mathrm{HCC}$ in patients with nodules detected during surveillance US (3). The AASLD HCC diagnostic algorithm initially included CEUS with CT and MRI, but the CEUS was removed from the guidelines in 2010. This was due to the possibility of misdiagnosing intrahepatic cholangiocarcinoma (ICC) as HCC and was based on one retrospective study [4]. Since then, there have been multiple studies investigating the accuracy of CEUS for focal liver lesion characterization. These studies have shown ICC often show arterial phase rim enhancement, unusual for HCC, and washout which tends to be much earlier than that of HCC, and of marked degree [5, 6]. As a result, the most recent 2017 AASLD guidelines for the treatment of hepatocellular carcinoma acknowledged, that although not used widely in North America, multiphasic contrast-enhanced ultrasound (CEUS) also can be used to diagnose HCC noninvasively, but further studies are needed (3).

Imaging diagnosis of HCC can be challenging, especially in patients with advanced cirrhosis, in which structural and physiological alterations of the background liver parenchyma can impair HCC detection and diagnosis (7). It is also complicated by the variety of regenerative and dysplastic nodules seen in patients with cirrhosis $(8,9,10,11)$. Furthermore, early HCC exhibits minimal neovascularization and typically does not manifest arterial hypervascularity at imaging, making its diagnosis challenging $(12,13)$. In addition to the full spectrum of hepatocellular nodules, other nodules, such as hemangiomas, cysts, intrahepatic cholangiocarcinoma (ICC), metastases, as well as pseudo-lesions, such as confluent fibrosis, perfusion alterations, focal fat deposition and sparing, further complicate liver imaging $(14,15)$.

Patients with chronic liver disease are at risk for both HCC and intrahepatic cholangiocellular carcinoma (ICC). Since ICC precludes transplantation, making differentiation between these tumors is critically important. Multiple investigators studied the differences in CEUS appearance of ICC and HCC with similar results $(16,17,18,19)$. Both HCC and ICC demonstrate similar hyper-enhancement in the arterial phase on CEUS, but show drastically different behavior on the delayed phase CEUS imaging with ICC washing out much sooner (typically $<60 \mathrm{sec}$ after contrast injection) and to a greater degree compared to HCC, which tend to wash-out late (>60 sec after contrast injection) and demonstrate mild washout degree. Han et al. demonstrated that at 3 min after injection contrast washout was detected in all ICCs, but in only $12.5 \%$ of HCC tumors ( $<<0.001$ ) (16). They concluded that majority of HCCs can be characterized by observation of no or delayed weak washout. In contrast, more rapid washout was characteristic of ICC (16). The authors also noted that there were no significant difference in the degree of ICC washout between patients with chronic hepatitis and those without. Li et al. demonstrated no difference between ICC and HCC appearance in the arterial phase of CEUS imaging (17). However, similar to other studies, ICC demonstrated earlier washout onset compared to HCC ( 47.9 s vs. 90.9 s respectively). When the authors combined early washout onset and marked washout degree as diagnostic criterion for ICC, the CEUS demonstrated 78.8\% sensitivity, $88.0 \%$ specificity, $81.3 \%$ PPV, $86.3 \mathrm{NPV}$ and $84.3 \%$ diagnostic accuracy. Significant differences in timing and degree of contrast wash-out on CEUS between ICC and HCC were also confirmed in two large studies by Wildner D et al. $(18,19)$. 
In order to improve liver imaging in patients at risk for $\mathrm{HCC}$, an expert panel of the American College of Radiology (ACR) introduced the "Liver Imaging Reporting and Data System" (LI-RADS), a system of standardized terminology and criteria for interpretation and reporting of liver observations in livers at risk for HCC (20). LI-RADS uses a diagnostic algorithm, based on a lexicon of precisely defined terms and imaging features, to assist radiologists in assigning the probability of HCC to nodules visualized in the at risk liver. Originally developed for CT and MRI, LI-RADS has been extended in 2016 to include CEUS $(21,22,23)$. LI-RADS was developed based on evidence, expert opinion and a desire to be consistent with the other existing diagnostic systems in the United States, namely, the imaging criteria embedded in the AASLD HCC clinical practice guidelines and the imaging criteria included in the Organ Procurement and Transplantation Network (OPTN) transplant policy $(24,25)$.

CEUS can significantly improve liver imaging in patients at risk for HCC development. Ultrasound imaging is portable, provides real-time imaging with high temporal and spatial resolution and uses no ionizing radiation $(26,27,28)$. After detection of a new liver nodule on surveillance US, there is a potential for immediate characterization with CEUS instead of scheduling a CT and MRI at a later time or date. In patients at risk for HCC, CEUS has specificity and sensitivity for HCC diagnosis comparable to CT and MRI (29). It also is less expensive than CT and MRI, and is more readily available worldwide. Ultrasound contrast agents are extremely well tolerated and demonstrate no nephrotoxicity, making them exceptionally safe $(30,31,32,33)$. CEUS of the liver becomes even more relevant with the recent FDA approval of a second-generation ultrasound contrast agent Lumason (Bracco Diagnostics Inc., Monroe Township, NJ) for liver imaging, making focal liver lesion characterization with CEUS the obvious next step after lesion detection on surveillance ultrasound.

This manuscript was prepared to provide readers with detailed description of CEUS technique and instrumentation used for CEUS LI-RADS focal liver nodule characterization in patients at risk for $\mathrm{HCC}$ and to provide a uniform lexicon to be used for imaging findings reporting.

\section{CEUS LI-RADS}

CEUS LI-RADS currently includes 8 categories characterizing focal liver observations based on HCC probability, from definitely benign (LR-1) to definitely HCC (LR-5), and probably or definitely malignant, but not necessarily HCC (LR-M). It also includes LR-NC category for observations that are Not Categorized due to image degradation or omission and LR-TIV category for observations with Tumor In Vein.

The details of categories, algorithm, major features, and ancillary features can be downloaded from the ACR website (https://www.acr.org/Quality-Safety/Resources/ LIRADS). 


\section{Indications for CEUS}

Although the entire liver is evaluated in its entirety on the B-mode scan to determine if there are observations or nodules that should be evaluated with CEUS, generally two to three nodules represents the maximum that can be thoroughly characterized in a CEUS examination. Further, if HCC or malignancy is shown on CEUS, staging the disease with CT or MR scan prior to treatment is usually recommended. CEUS is also excellent for evaluating specific nodules or problems that have previously been identified on either CT or MR imaging (34).

In patients at risk for HCC, CEUS can be used for the following indications:

Assess nodules $\geq 10 \mathrm{~mm}$ detected on surveillance US.

Assess LR-3, LR-4, and LR-M observations detected on prior CT or MRI.

Detect arterial phase hyperenhancement (APHE) when mistiming is suspected as the reason for its absence on prior CT or MRI.

Assess biopsied observations with inconclusive histology.

Guide biopsy or treatment of observations difficult to visualize with B-mode US.

Help select appropriate observation(s) or observation component(s) for biopsy.

Monitor changes in enhancement pattern over time for selected LR-3 or LR-4 observations.

Differentiate tumor in vein ("tumor thrombus") from bland thrombus.

To assess treatment response (not addressed in the CEUS LI-RADS v2016 and v2017).

CEUS is most reliable when assessing nodules $\geq 10 \mathrm{~mm}$ visible on pre-contrast B-mode images. In select cases, expert CEUS examiners, at their discretion, can perform CEUS to characterize smaller nodules or nodules occult on pre-contrast B-mode ultrasound using anatomical landmarks, image fusion or repeat contrast injections. Such characterization requires substantial experience and expertise. It is outside the purview of CEUS LI-RADS v2016 and v2017. Both treatment response assessment and imaging of observations not visible on pre-contrast B-mode may be addressed in CEUS LI-RADS v2020.

\section{Technical Recommendations}

\section{Examiner Training}

Appropriate ultrasound examiner and reader training are of paramount importance for successful CEUS implementation. Currently, the most robust training guidelines on CEUS are those developed by the EFSUMB (35). They define three levels of training and recommend that CEUS should be performed by operators at Level 2 competence level or higher, who is able to:

Perform a thorough CEUS examination of the liver including documentation and appropriate cine loop storage during all relevant contrast enhancement phases 
Recognize focal lesions and vascular disorders and be proficient in identifying appropriately the conditions for CEUS investigation

Recognize effect of treatment by CEUS

Write an appropriate report

Critically compare CEUS findings to other imaging modalities (CT, MRI, PET)

The practice of CEUS also requires knowledge of ultrasound contrast agent administration and contraindications and the skill to handle possible side effects, such as allergic and cardiopulmonary reactions.

\section{CEUS imaging}

Equipment-Ultrasound scanners equipped with appropriate software and hardware packages for contrast-enhanced imaging must be used. CEUS imaging is performed by separating signal from soft tissues that exhibit linear resonance under insonation from microbubbles, which are characterized by significant amount of non-linear resonance under the same conditions. These two signals (linear US signals from soft tissues and non-linear US signals from microbubbles) can be separated using various, often proprietary combinations of pulse inversion, frequency and amplitude modulation. As a result, modern ultrasound scanners allow near perfect soft tissue cancelation in the contrast-specific imaging mode to produce predominantly-microbubble images. This successful soft tissue cancelation will produce near complete disappearance (black screen) of soft tissue signal in the CEUS window, which is normal without presence of intravenous microbubbles and should not be mistaken for system malfunction (Figure 1).

To minimize the disruption of the microbubbles, CEUS imaging is performed at low acoustic pressures with mechanical index (MI) ranging from 0.05 to 0.3 . For comparison, routine ultrasound B-mode imaging is performed with MI greater than 1 and Doppler ultrasound with even higher levels (usually around MI 1.4), which would be detrimental for microbubbles. In addition to decreasing microbubble destruction, low MI imaging is helpful to reduce unwanted tissue harmonics, facilitating clear detection of contrast signals and improving signal-to noise ratio.

\section{Contrast Agents}

Currently, there are two purely intravascular ultrasound contrast agents with proven track record in liver imaging available for clinical use in the USA.

Lumason (sulfur hexafluoride lipid-type A microspheres, Bracco Diagnostics Inc., Monroe Township, NJ). This agent is marketed as SonoVue outside of the USA. The agent is supplied as a vial with $25 \mathrm{mg}$ of lipid-type A lyophilized powder and headspace fill of $60.7 \mathrm{mg}$ sulfur hexafluoride. It is reconstituted with $5 \mathrm{~mL}$ Sodium Chloride $0.9 \%$ and can be used for several hours after the reconstitution. This agent is approved by the FDA for liver imaging in adults and pediatric patients.

Definity (Perflutren Lipid Microsphere, Lantheus Medical Imaging Inc., N. Billerica, MA). This agent is supplied as a single 2-mL clear glass vial containing a lipid blend 
and $6.52 \mathrm{mg} / \mathrm{mL}$ octafluoropropane in the headspace. Definity vials are activated by shaking for 45 seconds using a proprietary VIALMIX activation device. This agent is approved by the FDA for use in echocardiography in the USA. Liver imaging with Definity is considered off-label in the USA, but can be performed, if medically indicated.

Another purely intravascular agent Optison (Perflutren Protein-Type A Microspheres Injectable Suspension, GE Healthcare, Princeton, NJ) is available for clinical use in the USA, but the documentation of successful use of this agent for liver imaging is lacking.

It should be noted that the current version of CEUS LI-RADS is based on use of purely intravascular contrast agents listed above. Use of the newer contrast agent Sonazoid (perflubutane microbubbles within a phosphatidyl serine shell, GE Healthcare, Oslo) is currently limited to Japan, South Korea, Denmark and Norway where this agent is approved for clinical use. This agent demonstrates prolonged liver uptake due to increased stability of the shell and active phagocytosis by Kupffer cells. It has a potential of significantly improving diagnostic accuracy of CEUS and its use may be addressed in CEUS LI-RADS v2020.

\section{Safety considerations}

Microbubble contrast agents are generally well-tolerated with only rare adverse events, as shown by nearly two decades of experience. Ultrasound contrast agents have no known renal toxicity in approved doses (36). Large post-marketing studies performed in response to an FDA black box warning placed in 2007, confirmed a very low rate of adverse effects $(37,38$, 39). This led to substantial softening of contraindications and removal of a previously mandated 30 minute monitoring period after administration of microbubble contrast agent.

A large retrospective multi-center study of the safety of Definity (66,164 doses) and Optison (12,219 doses), including over 10,000 doses to critically ill patients, showed severe allergic reactions in only 8 patients ( $0.01 \%$ incidence) and allergoid reactions in only 4 patients (0.006\% incidence) (40). Another large retrospective study of 16,025 patients receiving Definity or Optison demonstrated overall adverse effect rate of $0.12 \%$ and the incidence of serious events (with no fatalities) of $0.031 \%$ (41).

Safety data for Lumason is gleaned from post-marketing surveillance and clinical experience with SonoVue, the name by which Lumason is marketed outside the United States and has been available since 2001. In post-marketing surveillance of 1,651,451 patients exposed to SonoVue from April 1, 2001 through December 31, 2010, 217 serious adverse events were reported $(0.013 \%$ incidence), with 162 being allergy-like or anaphyllactoid $(0.0098 \%$ incidence) (42).

Even though adverse reactions are extremely rare, similar to CT and MRI contrast agents, all microbubble injections should be performed in appropriate settings and supervised by a board certified physician. Resuscitation equipment and trained personnel should be immediately available to the patient during each CEUS exam (43). Allergic reactions to ultrasound contrast agent they should be managed in accordance to standard contrast guidelines as described in the ACR Manual on Contrast Media (36). 


\section{Patient positioning and precontrast examination}

Patients should first undergo baseline US imaging to identify the observation/nodule and select the appropriate acoustic window for CEUS. Generally, the patient is positioned supine with the right arm in abduction. Having the sonographer/physician seated makes performing the exam easier and introduces less motion. The transducer is positioned in an approach whereby pre-contrast B-mode imaging best defines the lesion(s). Intercostal imaging may be the preferable approach for many lesions. As a general guide, it is often better to image in the longitudinal (sagittal or coronal) plane because this keeps the movement from breathing within the scan plane. Whatever the chosen approach, it should allow the target liver observation to be continuously visible during scanning. After appropriated patient position is established and acoustic window selected, B-mode images and measurements of observation, as well as sweeps through target focal liver observation in the transverse and sagittal planes should be acquired.

\section{Contrast agent administration}

After satisfactory patient positioning is achieved and pre-contrast imaging performed, the microbubble contrast agent bolus is injected intravenously.

Contrast agent dose-Recommended dose of ultrasound contrast agents for liver imaging is $2.4 \mathrm{ml}$ of Lumason or $0.2 \mathrm{ml}$ of Definity. There are several factors that could influence dose of ultrasound contrast for a particular examination. In patients with low BMI, dose of contrast could be decreased by 20-25\% to avoid over-enhancement of the liver, which complicates detection of mild contrast washout. Imaging of very superficial liver lesions with higher frequency probes will require contrast dose increase up to $4.8 \mathrm{~mL}$ of Lumason or $0.4 \mathrm{ml}$ of Definity. In addition to patient factors, the contrast dose could be adjusted based on the sensitivity of the equipment used for CEUS examination. However, these contrast dose adjustments are infrequent and default doses of $2.4 \mathrm{ml}$ of Lumason or 0.2 $\mathrm{ml}$ of Definity will provide appropriate contrast enhancement in the majority of patients.

Contrast agent injection-Contrast injection should be performed through an IV line, preferably 20G, to avoid bubble destruction. Injection through central venous lines and infusion ports is acceptable as long as all safety and aseptic requirements are met. Use of the central venous lines and infusion ports will shorten the contrast arrival time. Contrast bolus should be delivered over 2-3 seconds. Care should be taken to prevent increase in contrast syringe pressure, since this can destroy the microbubbles within the syringe, leading to reduced enhancement and impaired image quality. The bolus of contrast should be immediately followed by a 5-10 $\mathrm{mL}$ normal saline flush delivered at the rate of approximately $2 \mathrm{~mL} / \mathrm{sec}$.

Contrast timer-If the contrast injection is performed into IV line extension, the scanner's contrast timer is usually started at the end of contrast injection (immediately prior to or simultaneous with onset of saline flush). However, if the contrast injection is performed directly into the peripheral IV catheter, the scanner's contrast timer is usually started at the beginning of contrast bolus injection. Regardless of the IV setup, start of the contrast timer should be kept consistent to avoid mistiming of contrast washout. 


\section{Suggested imaging parameters}

Imaging should be performed in a dual screen format showing a low MI B-mode image alongside the contrast-only display to provide anatomic guidance and to ensure that target lesion is kept within the field of view during CEUS. One of the drawbacks of the dual screen technique is that both B-mode and contrast images are created using same low MI technique, resulting in significant degradation of B-mode image quality. Alternatively, CEUS imaging could be performed in contrast-only mode or using contrast overlay on top of B-mode images. These methods are rarely used in clinical practice and could complicate analysis of CEUS images.

Users should refer to the ultrasound scanner manufacturer manuals and, if needed, obtain additional technical support from the manufacturers, to ensure proper system settings before undertaking CEUS studies.

\section{Transducer selection}

Selecting an appropriate ultrasound transducer is critically important for successful CEUS examination. The transducer choice is usually based on the depth of the examined lesion and the characteristics of the acoustic window. Although most ultrasound scanners have multiple transducers that can be used for liver imaging, not all of them will support low MI contrastspecific imaging. The majority of US scanners have it enabled on curved array probes operating between 1 and $9 \mathrm{MHz}$ and on some higher-frequency linear transducers. It should be noted that all clinical ultrasound contrast agent are optimized to resonate at relatively low frequencies and the use of the higher frequency transducers require increase in contrast agent dose, which is usually doubled for imaging with linear transducers. As a general rule, CEUS liver imaging should be performed with a curved array transducer at a frequency of around 3-4 MHz to maximize contrast signals, with higher frequency linear transducers reserved for small superficial lesions.

\section{Depth of penetration and focal zone}

Depth of penetration of CEUS is typically less than B-mode imaging due to increased microbubble scatter of and increased signal attenuation from the microbubbles in the near field (self-shadowing) and low MI used for contrast specific imaging. To achieve the most optimal results, the acoustic window should be carefully selected allowing the examined lesion to be scanned as close to the transducer as possible, maintaining approximately $2 \mathrm{~cm}$ distance from the transducer to minimize potential image degradation from excessive microbubble destruction in the near field.

Appropriate placement of the focal zone is another very important step to ensure the high quality of CEUS imaging. To achieve an appropriate balance between near-field contrast destruction and appropriate penetration, the focal zone should be positioned just deep to the target lesion for most ultrasound scanners (Figure 2B). However, deeper focal zone placement might be used to achieve a more uniform acoustic field, which improves sensitivity to the contrast agents (Figure 2C). Positioning focal zone very far from the transducer will generally require an increase of MI to improve signal intensity and depth of penetration. This will result in increased destruction of the microbubbles in the near field, 
degrading near field image quality and occasionally imitating contrast wash-out in the late phase. Positioning the focal zone too close to the transducer will generally result in a decrease of MI to preserve microbubbles from excessive degradation in the near field (Figure 2D). As a result, the overall signal intensity and depth of penetration will be decreased, resulting in significant signal loss in deeper regions.

\section{Gain}

Gain settings allow the examiner to adjust both B-mode and CEUS signal amplification. In general, results of gain changes in CEUS are similar to the effects seen in Color or Power Doppler modes. Setting gain too low will result in significant signal loss (Figure 2E). Setting gain too high will increase nonspecific image noise, which appears as random signals distributed diffusely over the entire image field resulting in pseudo-enhancement (Figure $2 \mathrm{~F})$. The gain setting should be set as high as possible providing adequate soft tissue suppression without displaying pseudo-enhancement in the liver parenchyma, so the contrast mode image looks almost devoid of signal in preparation for contrast arrival. After the arrival of contrast, image gain can be adjusted accordingly. It should be noted that with some ultrasound scanners, it is normal to observe some artifacts produced by strong reflectors in the field of view, such as skin, muscle and liver capsule and large vessels (Figure 1). This static pseudo-enhancement is usually easily recognizable and should not be suppressed by further decrease in CEUS image gain.

\section{Dual/simultaneous calipers}

Availability of simultaneous caliper display on both screens is ideal for observation/nodule localization, especially in situations where the lesion is not very obvious on B-mode images or to confirm hyperenhancement or washout is co-localizing with the observation/nodule (Figure 3). This will serve to guide the operator's eye to arrival of contrast in the field of view, and to assess contrast enhancement, particularly of smaller and less obvious observations (i.e. isoenhancing or heterogeneously enhancing observations or nodules).

\section{Imaging protocol}

One of the great advantages of CEUS over CT and MRI is real time imaging and this benefit must be used to its fullest. It should be noted; however, that continuous insonation of large portions of highly vascular tissues (i.e. liver) may result in excessive destruction of microbubbles and substantial decrease in the degree of contrast enhancement in the late phase. To maximize benefits of real-time CEUS imaging and to preserve enough microbubbles to allow late contrast washout detection, we recommend the following imaging strategy (Figure 4).

Imaging should be performed continuously from contrast injection until peak AP enhancement to capture peak AP enhancement and characterize the presence, intensity (hyper, iso, or hypo) and pattern of AP enhancement (diffuse, mosaic, rim, peripheral globular). Alternatively, continuous imaging could be extended beyond peak AP enhancement until $60 \mathrm{sec}$ after contrast injection to determine presence of early washout. After 60 seconds, imaging should be performed intermittently (5-10 sec every 30-60 sec) to detect late washout and assess its degree. This technique will minimize microbubble 
destruction until microbubbles are cleared completely from the circulation (4-6 min after injection) and improve the ability to detect late washout and assess its degree.

Image recording should be performed continuously from first bubble arrival through peak AP enhancement as a minimum requirement. Optionally the cine loop can be continued beyond the AP enhancement peak until 60 seconds after injection. After 60 seconds, recording of static images at 60 seconds and with every intermittent (every 30-60sec) acquisition thereafter will be sufficient to document and evaluate the presence, timing, and degree of washout.

Sweeping the entire liver in the late phase can help to identify additional areas of washout as focal hypo-enhancing regions in the liver. However, in patients with advanced cirrhosis, the sensitivity of this approach may be compromised due to relatively poor background liver enhancement. If the area of washout is associated with a solid nodule visible on B-mode imaging, this nodule can be further characterized with an additional injection of contrast. If the area of washout is not associated with an identifiable nodule on B-mode ultrasound, radiologists/sonologists may at their discretion either proceed with further CEUS characterization or refer for an alternative diagnostic test. Repeat injection should be performed in accordance with contrast manufacturer guidelines. The maximum total contrast dose should not exceed that listed on the contrast agent package insert: $4.8 \mathrm{ml}$ of Lumason or 20 microL/kg of the activated Definity $(44,45)$. Please, refer to the specific contrast agent package insert for the most recent dosing information.

\section{Patients with multiple liver nodules}

In general, up to two to three nodules can be successfully imaged in one patient. Imaging of multiple nodules often requires multiple injections and careful planning of patient positioning to maximize the use of limited acoustic windows. To accelerate microbubble clearance from the circulation between injections, the liver and the spleen may be briefly insonated with high MI (such as used in conventional B-mode/color Doppler imaging). This approach will significantly improve visualization of arterial phase enhancement on subsequent injections by eliminating free circulating microbubbles from the prior injection.

\section{Potential technical pitfalls and challenges}

One of the most important CEUS limitations is the inability to image the entire liver in the arterial phase, since only a part of the organ, usually containing the region of interest with focal liver observation visible on the pre-contrast B-mode can be imaged with each injection. This limitation can be addressed by careful scanning of the entire liver in the late phase to detect focal areas of wash-out, which can be further evaluated in the arterial phase with repeat contrast injections.

Some small lesions, usually less than $10 \mathrm{~mm}$ or lesions with subdiaphragmatic or very deep location can be challenging to visualize on CEUS. As a basic rule, if the lesion is difficult to clearly identify on pre-contrast B-mode imaging, it might be challenging to produce highquality CEUS images. CEUS might also be limited in patients with high BMI and in the patients with severe hepatic steatosis, mainly due to substantial signal attenuation. In some patients, interfering bowel gas might limit lesion visualization. 
Nonlinear artifact, a CEUS phenomenon associated with pseudo-enhancement following ultrasound contrast agent injection, could also complicate CEUS imaging. It is caused by the nonlinear propagation of sound through intervening microbubble-perfused tissue and is, therefore, most marked in hyperechoic objects in deep regions. Its original description is related to potential pseudo-enhancement of echogenic appearing treatment sites on CEUS following radiofrequency ablation $(46,47,48)$.

\section{Lexicon of Imaging Findings}

Each CEUS study should be evaluated using the following criteria:

\section{Adequacy of CEUS exam}

The CEUS examination will be characterized as adequate when the entire region of interest, an observation or nodule can be unequivocally visualized for the entirety of the exam. Observations that cannot be categorized due to image degradation or omission are characterized as CEUS LR-NC (Not Categorizable).

\section{Observation}

A distinctive area with imaging features that differ from those of adjacent liver parenchyma. It may be a nodule or pseudolesion.

Nodule

A discrete mass of rounded or irregular shape. It should be noted, that CT and MRI LIRADS use term "observations" to describe focal signal abnormalities. This is because many findings detected on CT and MRI may not represent true nodules. On CEUS, with exception of focal fatty infiltration and sparing, most of the focal liver observations are true nodules.

\section{Observation location}

Liver observation location using the Couinaud liver segment classification.

\section{Observation size}

The largest dimensions of the liver observation reported in three perpendicular planes, preferably on precontrast B-mode imaging. If observation margins are indistinct on B-mode imaging, measurements on the arterial phase CEUS images could be performed instead. It should be noted that CEUS measurements could overestimate true size of the observation, since it might be affected by contrast blooming artifact or inclusion of hyperenhancing surrounding liver parenchyma in the measurement.

\section{Unequivocal diameter increase}

Increase in the maximal diameter of an observation, measured on exams performed on different dates, which is not attributable to artifact, differences in technique or modality between the two exams, or measurement error. CEUS LI-RADS uses it as an ancillary feature to suggest malignancy. 


\section{Unequivocal diameter decrease}

Unequivocal decrease in the maximal visualized diameter of an observation, measured on exams performed on different dates, which is not attributable to artifact, differences in technique or modality between the two exams, or measurement error. CEUS LI-RADS uses it as an ancillary feature to suggest benignity.

- $\quad$ Note: Currently there are no universally accepted guidelines for tumor measurements on ultrasound. Therefore, we rely on the judgment to the ultrasound examiner to determine if changes in size are unequivocal (i.e. not attributable to artifact, differences in technique or modality between the two exams, or measurement error).

\section{Diameter stability}

No significant change in maximal diameter of an observation, measured on exams performed on different dates over $\geq 2$ years and in the absence of treatment. CEUS LI-RADS uses it as an ancillary feature to suggest benignity.

\section{Enhancement phases}

The dual blood supply of the liver from the hepatic artery (25\%-30\%) and the portal vein (70\%-75\%) gives rise to three overlapping vascular phases on CEUS study. While we use these enhancement phases in general terms, it is very important for CEUS evaluation to monitor the timing of washout in "seconds" as explained in washout features to accurately differentiate HCC from potential other malignancy. This is performed by having a contrast timer on the US screen which will allow for precise timing of any event during the CEUS examination.

- The hepatic arterial phase usually starts within 10-20 seconds after injection and continues to $30-45$ seconds, depending on the individual's circulatory status. It provides information on the degree and pattern of the arterial vascular supply. This phase may occur very rapidly and the real-time nature of CEUS is needed to capture the essential features of the enhancement pattern, often best seen in a slow replay of a stored cine loop (Figure 5).

- The portal venous phase usually begins at about 30-45 seconds and lasts until 2 minutes after injection.

- $\quad$ The late phase usually starts at about 2 min after contrast injection and lasts until the clearance of the microbubbles from the circulation (4-6 minutes after contrast injection).

\section{Arterial phase enhancement}

The arterial phase enhancement of a liver nodule is assessed by comparing the intensity of the signal from a liver nodule with the signal from adjacent liver at the same depth, as evaluated on continuous image acquisition from the arrival of the first bubble to the peak of arterial phase enhancement. 
- $\quad$ The degree of arterial phase enhancement should be characterized using four categories, as follows:

- $\quad$ Hyperenhancement: higher contrast agent signal intensity in the liver nodule as compared with the intensity of the adjacent liver at the same depth (Figure 6). This feature can be considered present if it is demonstrated in either the entire nodule or in only a portion of the nodule.

- $\quad$ Isoenhancement: equivalent contrast agent signal intensity in the liver nodule as compared with the adjacent liver at the same depth (Figure 7).

- $\quad$ Hypoenhancement: less contrast agent signal intensity in the liver nodule as compared with the adjacent liver at the same depth (Figure 8).

- $\quad$ No-enhancement: lack of contrast agent signal in the liver nodule (Figure 9).

- The pattern of arterial phase enhancement should be characterized using four categories, as follows:

- $\quad$ Enhancement in whole: enhancement of the entire nodule as compared to the surrounding liver parenchyma at the same depth (Figure 6)

- $\quad$ Nodule-in-nodule enhancement. Increased enhancement of one or more inner nodules within a larger enhancing nodule (Figure 10). CEUS LIRADS uses it as an ancillary feature to suggest malignancy, favoring HCC in particular.

- $\quad$ Rim-enhancement: enhancement mainly concentrated at the periphery of the liver nodule (Figure 11). This is usually followed by rapid and marked washout. This is a typical feature of metastatic lesions and liver nodules with rim enhancement should be characterized as CEUS LR-M. Careful measurements of the lesion including and excluding rim enhancement, and compared with the baseline image, if well seen, are beneficial to determine precise location of rim enhancement.

- Peripheral discontinuous globular enhancement: Typical feature of hemangiomas (CEUS LR-1) that demonstrate interrupted areas of increased enhancement along the periphery of the lesion in the early arterial phase. This phenomenon is temporary and is usually followed by centripetal progression of the enhancement and sustained enhancement in the late phase (Figure 12).

\section{Washout}

Visually assessed temporal reduction in contrast agent signal intensity of a nodule relative to adjacent liver following initial enhancement, resulting in hypoenhancement. At CEUS, all malignant nodules typically show washout - HCC, metastasis, intrahepatic cholangiocarcinoma (ICC), or hepatocholangiocarcinoma. Unlike CT or MRI with extracellular contrast agents, ICC and other fibrotic tumors do not show delayed central 
enhancement on CEUS. Washout is characterized by timing of washout onset and washout degree.

- Timing of washout onset. Timing of the first observation of washout reported in seconds after contrast bolus injection.

Early onset, detected < $60 \mathrm{sec}$ after contrast injection. CEUS LI-RADS uses early washout as a hallmark of CEUS LR-M lesions.

Late onset, detected $\geq 60 \mathrm{sec}$ after contrast injection. CEUS LI-RADS uses late washout as a hallmark of hepatocellular lesions.

- Washout degree, assessed by comparing degree of contrast enhancement in the nodule relative to surrounding liver.

Mild washout is used when the liver nodule becomes hypoechoic relative to the liver but continues to show some contrast enhancement (Figure 13). CEUS LIRADS uses mild washout that appears $\succeq 60 \mathrm{sec}$ after injection as a hallmark of hepatocellular lesions. Nodules initially demonstrating mild washout might eventually become virtually devoid of contrast ("punched-out"). If this occurs after 2 minutes, the classification does not alter.

Marked washout is used when the liver nodule becomes virtually devoid of contrast ("punched-out") within 2 minutes after contrast injection. (Figure 14). CEUS LI-RADS uses marked washout as a hallmark of CEUS LR-M lesions $\left({ }^{49}\right)$.

\section{Mosaic architecture}

Mosaic architecture refers to the presence within a mass of randomly distributed internal nodules differing in echogenisity on B-mode imaging and contrast enhancement intensity on CEUS. (Figure 15). This feature is characteristic of large HCCs and reflects the mosaic configuration with multiple confluent areas of tumor nodularity interspersed with fibrous septations, necrosis, hemorrhage, copper deposition, and fatty infiltration, as well as varying degrees of histologic differentiation (50). CEUS LI-RADS uses it as an ancillary feature to suggest malignancy, favoring $\mathrm{HCC}$ in particular.

\section{Tumor in vein}

Definite enhancing soft tissue in vein regardless of visualization of parenchymal mass/ nodule. It must have definite enhancement in the arterial phase followed by washout (regardless of onset or degree). (Figure 16).

- While not diagnostic of tumor in vein, features that may alert examiners to diagnosis include occluded or partially occluded vein with moderately to markedly expanded lumen, ill-defined or frankly disrupted walls or clear contiguity with a CEUS LR-5 nodule. By comparison, non-neoplastic bland thrombus does not enhance, usually does not expand the vein lumen to same degree and preserves the vein walls. 
- To reliably differentiate tumor in vein vs. partially occlusive/recanalized bland thrombus, careful assessment of the arrival time of contrast to the vein is needed:

- Early arrival of contrast material into the soft tissue in the vein at about the same time as opacification of hepatic arteries suggests tumor.

- $\quad$ Arrival of contrast material several ( 10) seconds after opacification of hepatic arteries favors venous flow around a non-occlusive bland thrombus.

- Confirmation of arterial wave flow on spectral Doppler may be of additional help in differentiating tumor within vein from non-occlusive bland thrombus.

- Tumor in peripheral portal veins may be mistaken for tumor nodules, erroneously downstaging the patient. Avoidance is facilitated by real-time imaging while sweeping through the liver, especially in the portal phase, to depict the tubular configuration of the tumor and its continuity with more central portal or hepatic veins.

\section{DISCUSSION}

To address the challenges of liver imaging in patients at risk for $\mathrm{HCC}$ and to provide a framework for assigning probability of $\mathrm{HCC}$ and other malignancy to imaging findings in patients at risk for HCC, the American College of Radiology developed LI-RADS, a system of standardized terminology and criteria for interpreting and reporting hepatic imaging findings in patients at risk for HCC. Initially designed for multiphasic CT and MRI it was recently extended to include CEUS. This manuscript represents a description of the technical aspects for CEUS and provides a detailed imaging protocol that should be used for uniformity of CEUS. It also provides a Lexicon of imaging findings that should improve accuracy of image analysis and reporting. We are confident that systematic use of CEUS LIRADS will decrease user-dependency of CEUS and provide a robust structured diagnostic and reporting framework that optimizes clinical use of CEUS in patients at risk for HCC.

\section{Acknowledgments}

The authors would like to dedicate this manuscript to our dear friend Dr. David Cosgrove (1938-2017). David was a thought leader in the clinical ultrasound and a passionate advocate of CEUS. He was one of the founding members of the CEUS LI-RADS Working Group and spent countless hours working on this project. His extraordinary knowledge, enthusiasm, and dedication were extremely valuable and will be greatly missed...

\section{References}

1. World Health Organization. GLOBOSCAN 2012: Estimated Cancer Incidence, Mortality and Prevalence Wordwide in 2017. http://globocan.iarc.fr/Pages/fact_sheets_cancer.aspx

2. Mitchell DG, Bruix J, Sherman M, Sirlin CB. LI-RADS (Liver Imaging Reporting and Data System): summary, discussion, and consensus of the LI-RADS Management Working Group and future directions. Hepatology. 2015; 61:1056-65. [PubMed: 25041904]

3. Heimbach J, Kulik LM, Finn R, Sirlin CB, Abecassis M, Roberts LR, Zhu A, Murad MH, Marrero J. AASLD guidelines for the treatment of hepatocellular carcinoma. Hepatology. 2017 Jan 28.doi: 10.1002/hep.29086 
4. Vilana R, Forner A, Bianchi L, Garcia-Criado A, Rimola J, de Lope CR, Reig M, Ayuso C, Bru C, Bruix J. Intrahepatic peripheral cholangiocarcinoma in cirrhosis patients may display a vascular pattern similar to hepatocellular carcinoma on contrast-enhanced ultrasound. Hepatology. 2010; 51(6):2020-2029. [PubMed: 20512990]

5. Li R, Yuan MX, Ma KS, Li XW, Tang CL, Zhang XH, Guo DY, Yan XC. Detailed analysis of temporal features on contrast enhanced ultrasound may help differentiate intrahepatic cholangiocarcinoma from hepatocellular carcinoma in cirrhosis. PLoS One. 2014; 9(5):e98612. [PubMed: 24874413]

6. Wildner D, Bernatik T, Greis C, Seitz K, Neurath MF, Strobel D. CEUS in hepatocellular carcinoma and intrahepatic cholangiocellular carcinoma in 320 patients - early or late washout matters: a subanalysis of the DEGUM multicenter trial. Ultraschall Med. 2015; 36(2):132-139. [PubMed: 25812115]

7. Ishak K, Baptista A, Bianchi L, Callea F, De Groote J, Gudat F, Denk H, Desmet V, Korb G, MacSween RN, et al. Histological grading and staging of chronic hepatitis. J Hepatol. 1995; 22:696-9. [PubMed: 7560864]

8. Park YN. Update on precursor and early lesions of hepatocellular carcinomas. Arch Pathol Lab Med. 2011; 135:704-715. [PubMed: 21631263]

9. Coleman WB. Mechanisms of human hepatocarcinogenesis. Curr Mol Med. 2003; 3:573-588. [PubMed: 14527088]

10. International Working Party. Terminology of nodular hepatocellular lesions. Hepatology. 1995; 22:983-993. [PubMed: 7657307]

11. Roskams T, Kojiro M. Pathology of early hepatocellular carcinoma: conventional and molecular diagnosis. Semin Liver Dis. 2010; 30:17-25. [PubMed: 20175030]

12. Trevisani F, Cantarini MC, Wands JR, Bernardi M. Recent advances in the natural history of hepatocellular carcinoma. Carcinogenesis. 2008; 29:1299-1305. [PubMed: 18515282]

13. Chou CT, Chou JM, Chang TA, Huang SF, Chen CB, Chen YL, Chen RC. Differentiation between dysplastic nodule and early-stage hepatocellular carcinoma: the utility of conventional MR imaging. World J Gastroenterol. 2013; 19:7433-7439. [PubMed: 24259975]

14. Jha RC, Mitchell DG, Weinreb JC, Santillan CS, Yeh BM, Francois R, Sirlin CB. LI-RADS categorization of benign and likely benign findings in patients at risk of hepatocellular carcinoma: a pictorial atlas. AJR Am J Roentgenol. 2014; 203:48-69.

15. Choi J-Y, Lee J-M, Sirlin CB. CT and MR imaging diagnosis and staging of hepatocellular carcinoma. Part II. Extracellular agents, hepatobiliary agents, and ancillary imaging features. Radiology. 2014; 273:30-50. [PubMed: 25247563]

16. Han J, Liu Y, Han F, et al. The degree of contrast washout on contrast-enhanced ultrasound in distinguishing intrahepatic cholangiocarcinoma from hepatocellular carcinoma. Ultrasound Med Biol. 2015; 41(12):3088-3095. [PubMed: 26386477]

17. Li R, Yuan MX, Ma KS, et al. Detailed analysis of temporal features on contrast enhanced ultrasound may help differentiate intrahepatic cholangiocarcinoma from hepatocellular carcinoma in cirrhosis. PLoS One. 2014; 9(5):e98612. [PubMed: 24874413]

18. Wildner D, Bernatik T, Greis C, et al. CEUS in hepatocellular carcinoma and intrahepatic cholangiocellular carcinoma in 320 patients - early or late washout matters: a subanalysis of the DEGUM multicenter trial. Ultraschall Med. 2015; 36(2):132-139. [PubMed: 25812115]

19. Wildner D, Pfeifer L, Goertz RS, et al. Dynamic contrast-enhanced ultrasound (DCE-US) for the characterization of hepatocellular carcinoma and cholangiocellular carcinoma. Ultraschall Med. 2014; 35(6):522-527. [PubMed: 25202903]

20. The Liver Imaging Reporting and Data System (LI-RADS). http://www.acr.org/quality-safety/ resources/LIRADS

21. Kono Y, Cosgrove D, Dietrich C, Jang HJ, Kim TK, Lyshchik A, Piscaglia F, Sirlin C, Willmann J, Wilson S. Contrast-Enhanced Ultrasound Liver Imaging Reporting and Data System for Diagnosis of Hepatocellular Carcinoma: Initial Proposal. J Ultrasound Med. 2016; 35(suppl):S1-S132.

22. Jang, HJ., Kono, Y., Sirlin, C., Lyshchik, A., Piscaglia, F., Cosgrove, D., Wilson, S. Incorporation of CEUS Into LI-RADS for Diagnosis of Hepatocellular Carcinoma (HCC): A Work in Progress. 
Radiological Society of North America Annual Meeting; 29 November - 4 December, 2015; Chicago, IL. GI212-ED-X

23. Piscaglia F, Wilson SR, Lyshchik A, Cosgrove D, Dietrich CF, Jang HJ, Kim TK, Salvatore V, Willmann JK, Sirlin CB, Kono Y. American College of Radiology Contrast Enhanced Ultrasound Liver Imaging Reporting and Data System (CEUS LI-RADS) for the diagnosis of Hepatocellular Carcinoma: a pictorial essay. Ultraschall Med. 2017; 38:320-324. [PubMed: 28329875]

24. Heimbach J, Kulik LM, Finn R, Sirlin CB, Abecassis M, Roberts LR, Zhu A, Murad MH, Marrero J. AASLD guidelines for the treatment of hepatocellular carcinoma. Hepatology. 2017 Jan 28.doi: 10.1002/hep.29086

25. OPTN Policy \#9: Allocation of Livers and Liver-Intestines. https://optn.transplant.hrsa.gov/media/ 1200/optn_policies.pdf

26. Claudon M, Dietrich CF, Choi BI, Cosgrove DO, Kudo M, Nolsøe CP, Piscaglia F, Wilson SR, Barr RG, Chammas MC, Chaubal NG, Chen MH, Clevert DA, Correas JM, Ding H, Forsberg F, Fowlkes JB, Gibson RN, Goldberg BB, Lassau N, Leen EL, Mattrey RF, Moriyasu F, Solbiati L, Weskott HP, Xu HX. World Federation for Ultrasound in Medicine; European Federation of Societies for Ultrasound. Guidelines and good clinical practice recommendations for Contrast Enhanced Ultrasound (CEUS) in the liver - update 2012: A WFUMB-EFSUMB initiative in cooperation with representatives of AFSUMB, AIUM, ASUM, FLAUS and ICUS. Ultrasound Med Biol. 2013; 39:187-210. [PubMed: 23137926]

27. Sawhney S, Wilson SR. Can Ultrasound With Contrast Enhancement Replace Nonenhanced Computed Tomography Scans in Patients With Contraindication to Computed Tomography Contrast Agents? Ultrasound Q. 2017; 33:125-132. [PubMed: 28141682]

28. Jang HJ, Kim TK, Burns PN, Wilson SR. CEUS: An essential component in a multimodality approach to small nodules in patients at high-risk for hepatocellular carcinoma. Eur J Radiol. 2015; 84:1623-35. [PubMed: 26092406]

29. Hanna RF, Miloushev VZ, Tang A, Finklestone LA, Brejt SZ, Sandhu RS, Santillan CS, Wolfson T, Gamst A, Sirlin CB. Comparative 13-year meta-analysis of the sensitivity and positive predictive value of ultrasound, CT, and MRI for detecting hepatocellular carcinoma. Abdom Radiol (NY). 2016; 41:71-9. [PubMed: 26830614]

30. Exuzides A, Main ML, Colby C, Grayburn PA, Feinstein SB, Goldman JH. A retrospective comparison of mortality in critically ill hospitalized patients undergoing echocardiography with and without an ultrasound contrast agent. JACC Cardiovasc Imaging. 2010; 3:578-85. [PubMed: 20541713]

31. Goldberg YH, Ginelli P, Siegel R, Ostfeld RJ, Schaefer M, Spevack DM. Administration of perflutren contrast agents during transthoracic echocardiography is not associated with a significant increase in acute mortality risk. Cardiology. 2012; 122(2):119-25. [PubMed: 22813648]

32. Aggeli C, Giannopoulos G, Roussakis G, Christoforatou E, Marinos G, Toli C, Pitsavos C, Stefanadis C. Safety of myocardial flash-contrast echocardiography in combination with dobutamine stress testing for the detection of ischaemia in 5250 studies. Heart. 2008; 94:1571-7. [PubMed: 18474538]

33. Parker JM, Weller MW, Feinstein LM, Adams RJ, Main ML, Grayburn PA, Cosgrove DO, Goldberg BA, Darge K, Nihoyannopoulos P, Wilson S, Monaghan M, Piscaglia F, Fowlkes B, Mathias W, Moriyasu F, Chammas MC, Greenbaum L, Feinstein SB. Safety of ultrasound contrast agents in patients with known or suspected cardiac shunts. Am J Cardiol. 2013; 112:1039-45. [PubMed: 23816393]

34. Jo PC, Jang HJ, Burns PN, Burak KW, Kim TK, Wilson SR. Integration of Contrast-enhanced US into a Multimodality Approach to Imaging of Nodules in a Cirrhotic Liver: How I Do It. Radiology. 2017; 282:317-331. [PubMed: 28099108]

35. Minimum training requirements for the practice of Medical Ultrasound in Europe. Ultraschall in Med. 2010; 31:426-427. [PubMed: 20725892]

36. ACR Manual on Contrast Media. https://www.acr.org/ /media/ 37D84428BF1D4E1B9A3A2918DA9E27A3.pdf 
37. Main ML, Hibberd MG, Ryan A, Lowe TJ, Miller P, Bhat G. Acute mortality in critically ill patients undergoing echocardiography with or without an ultrasound contrast agent. JACC Cardiovasc Imaging. 2014; 7:40-8. [PubMed: 24290568]

38. Parker JM, Weller MW, Feinstein LM, Adams RJ, Main ML, Grayburn PA, Cosgrove DO, Goldberg BA, Darge K, Nihoyannopoulos P, Wilson S, Monaghan M, Piscaglia F, Fowlkes B, Mathias W, Moriyasu F, Chammas MC, Greenbaum L, Feinstein SB. Safety of ultrasound contrast agents in patients with known or suspected cardiac shunts. Am J Cardiol. 2013; 112:1039-45. [PubMed: 23816393]

39. Main ML, Ryan AC, Davis TE, Albano MP, Kusnetzky LL, Hibberd M. Acute mortality in hospitalized patients undergoing echocardiography with and without an ultrasound contrast agent (multicenter registry results in 4,300,966 consecutive patients). Am J Cardiol. 2008; 102:1742-6. [PubMed: 19064035]

40. Wei K, Mulvagh SL, Carson L, Davidoff R, Gabriel R, Grimm RA, Wilson S, Fane L, Herzog CA, Zoghbi WA, Taylor R, Farrar M, Chaudhry FA, Porter TR, Irani W, Lang RM. The safety of deFinity and Optison for ultrasound image enhancement: a retrospective analysis of 78,383 administered contrast doses. J Am Soc Echocardiogr. 2008; 21:1202-6. [PubMed: 18848430]

41. Herzog CA. Incidence of adverse events associated with use of perflutren contrast agents for echocardiography. JAMA. 2008; 299:2023-5. [PubMed: 18460662]

42. https://www.fda.gov/downloads/AdvisoryCommittees/CommitteesMeetingMaterials/Drugs/ CardiovascularandRenalDrugsAdvisoryCommittee/UCM252880.pdf

43. Muskula PR, Main ML. Safety With Echocardiographic Contrast Agents. Circ Cardiovasc Imaging. 2017 Apr.10(4) pii: e005459. doi: 10.1161/CIRCIMAGING.116.005459

44. Lumason Prescribing Information. http://imaging.bracco.com/sites/braccoimaging.com/files/ technica_sheet_pdf/us-en-2017-01-04-spc-lumason.pdf

45. Definity Prescribing Information. http://www.definityimaging.com/pdf/ DEFINITY_US_PI_515987-0117.pdf

46. Yu H, Jang HJ, Kim TK, Khalili K, Williams R, Luek G, Hudson J, Burns PN. Pseudoenhancement within local ablation zone of hepatic tumors due to nonlinear artifact on contrastenhanced ultrasound. Am J Roentgenol. 2010; 194:653-659. [PubMed: 20173141]

47. Dietrich CF, Ignee A, Greis C, Cui XW, Schreiber-Dietrich DG, Hocke M. Artifacts and pitfalls in contrast-enhanced ultrasound of the liver. Ultraschall Med. 2014; 35(2):108-125. [PubMed: 24477558]

48. Li SY, Huang P, Cosgrove D, Xu H, Xu LL, Liang X, Cai XJ. Pseudoenhancement of Gallbladder Sludge: A Confusing Artifact Caused by Nonlinear Propagation of Ultrasound Through Microbubbles. Ultraschall Med. 2016 Jun; 37(3):307-9. [PubMed: 26882481]

49. Fowler KJ, Potretzke TA, Hope TA, Costa EA, Wilson SR. LI-RADS M (LR-M): definite or probable malignancy, not specific for hepatocellular carcinoma. Abdom Radiol (NY). 2017 Jun 3. [Epub ahead of print]. doi: 10.1007/s00261-017-1196-2

50. Hanna RF, Aguirre DA, Kased N, Emery SC, Peterson MR, Sirlin CB. Cirrhosis-associated hepatocellular nodules: correlation of histopathologic and MR imaging features. Radiographics. 2008; 28:747-69. [PubMed: 18480482] 


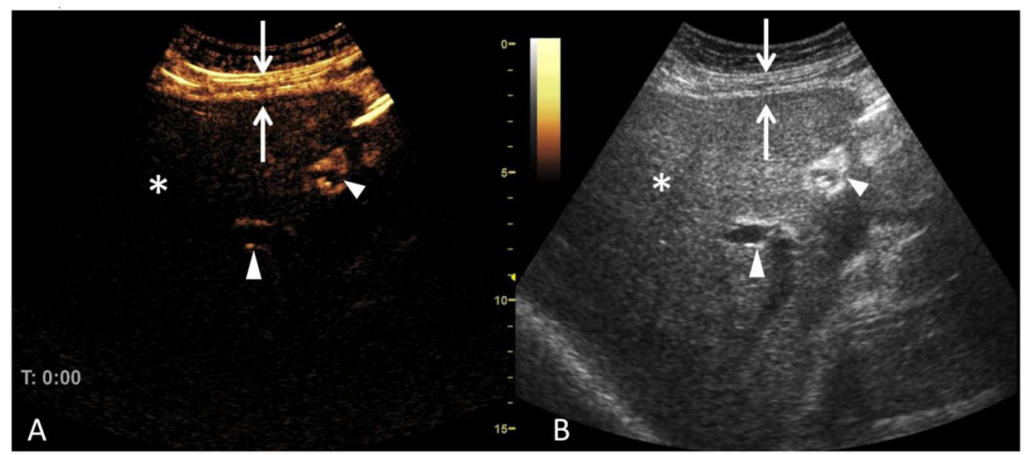

Figure 1.

Dual scree imaging with low MI B-mode image on the left and "black screen" CEUS image (A) before contrast administration. Please, note some amount of noise in the near field from strong reflectors such as skin, muscle and liver capsule (arrows) and large vessels (arrowheads) and complete removal of signal from the normal liver parenchyma (asterisk) visible on B-mode image (B). Contrast timer is seen at the bottom of the contrast screen. Image reproduced with permission from the ACR. 

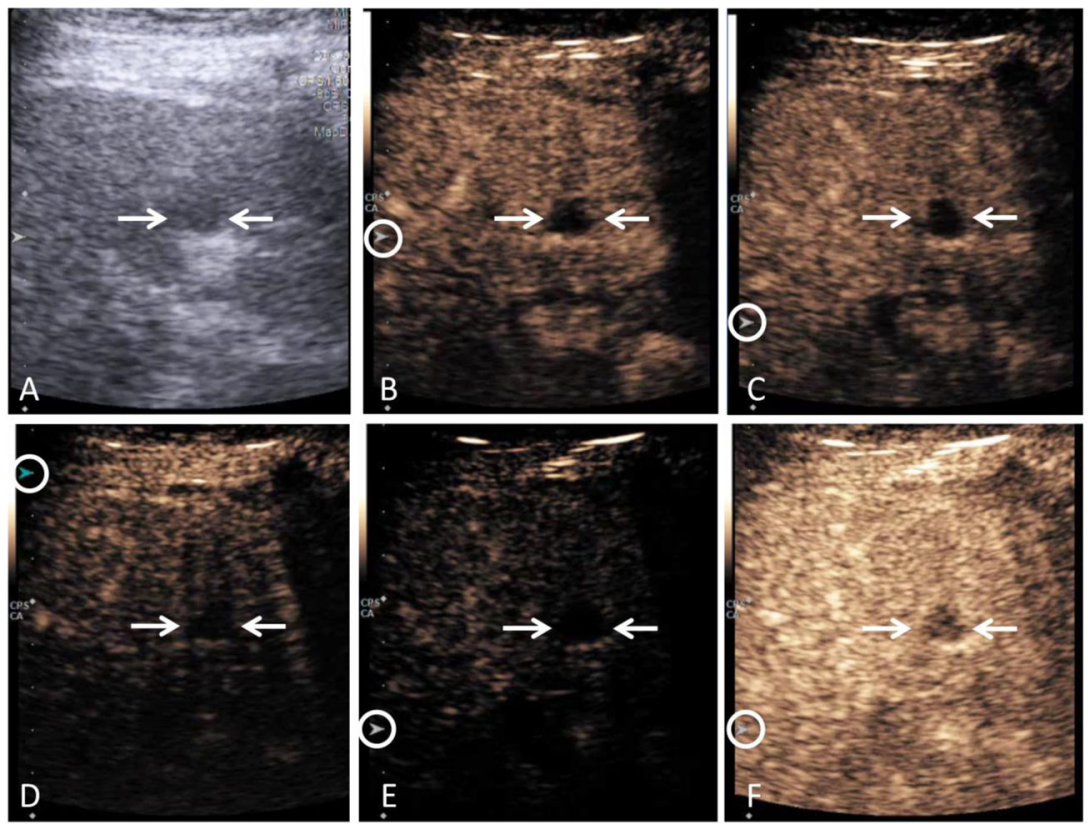

Figure 2.

CEUS focal zone and gain adjustments. B-mode image (A) demonstrates small indeterminate hypoechoic nodule (arrows). Focal zone adjustments: CEUS images with appropriate focal zone placement (circle) at the lower edge (B) or slightly deeper to the nodule (C) produced high quality CEUS images demonstrating nodule with absence of arterial phase enhancement, making it consistent with a cyst (LR-1). Also, note appropriate gain adjustment on both images $(\mathrm{B}, \mathrm{C})$. Positioning focal zone too close to the transducer (D) resulted in significantly decreased overall signal intensity and depth of penetration. Image gain adjustments: Setting CEUS gain too low (E), even with appropriately placed focal zone, resulted in significant signal loss. Setting gain too high $(F)$ increased nonspecific image noise and produced pseudo-enhancement in the liver cyst. Image reproduced with permission from the ACR. 


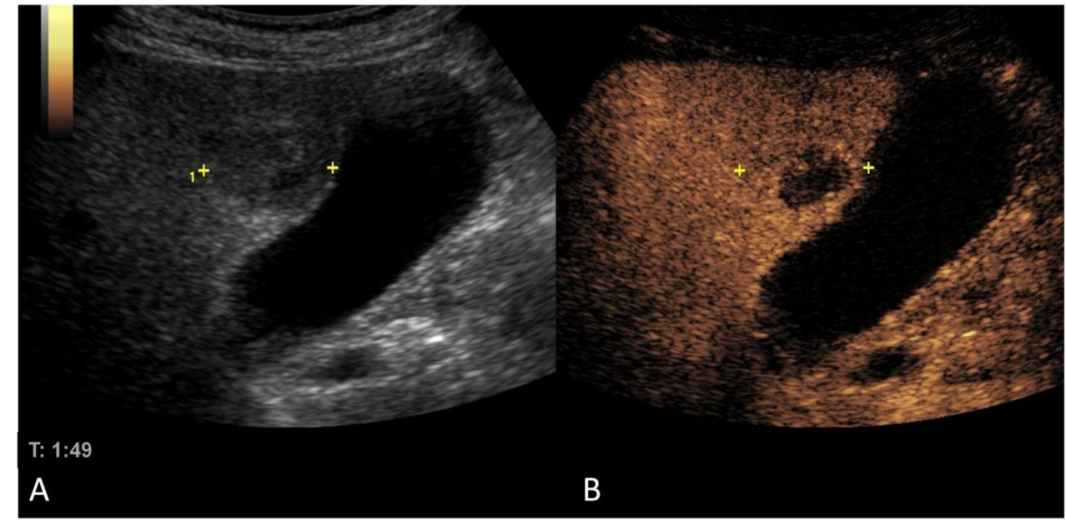

Figure 3.

Calipers placed on the edges of the lesion on B-mode image are automatically copied to CEUS image by the scanner, significantly improving lesion identification and characterization of enhancement pattern. Image reproduced with permission from the ACR. 


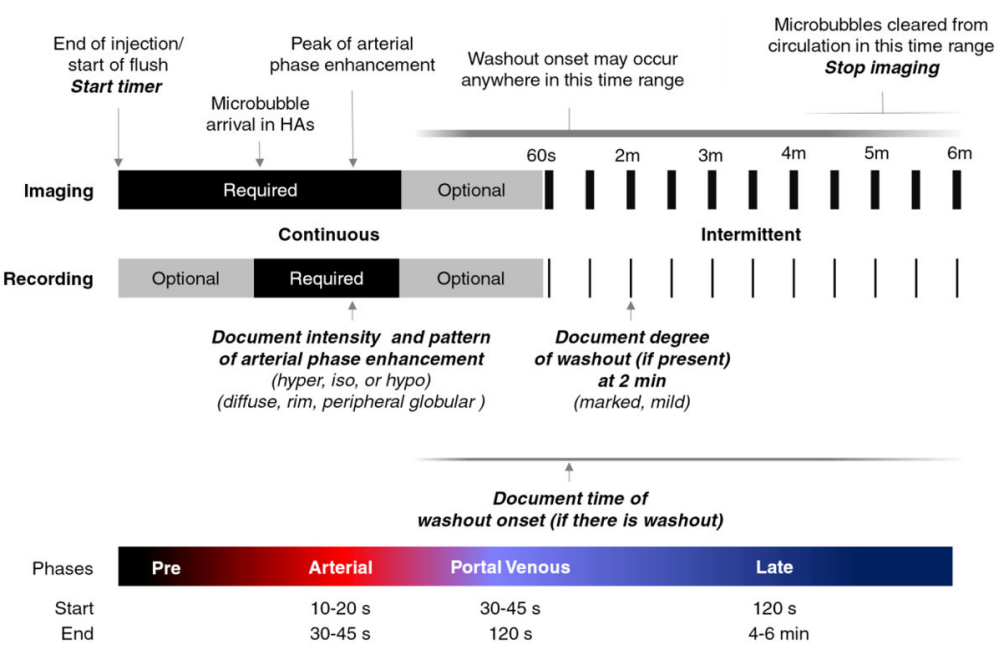

Figure 4.

Liver CEUS imaging protocol. Image reproduced with permission from the ACR. 

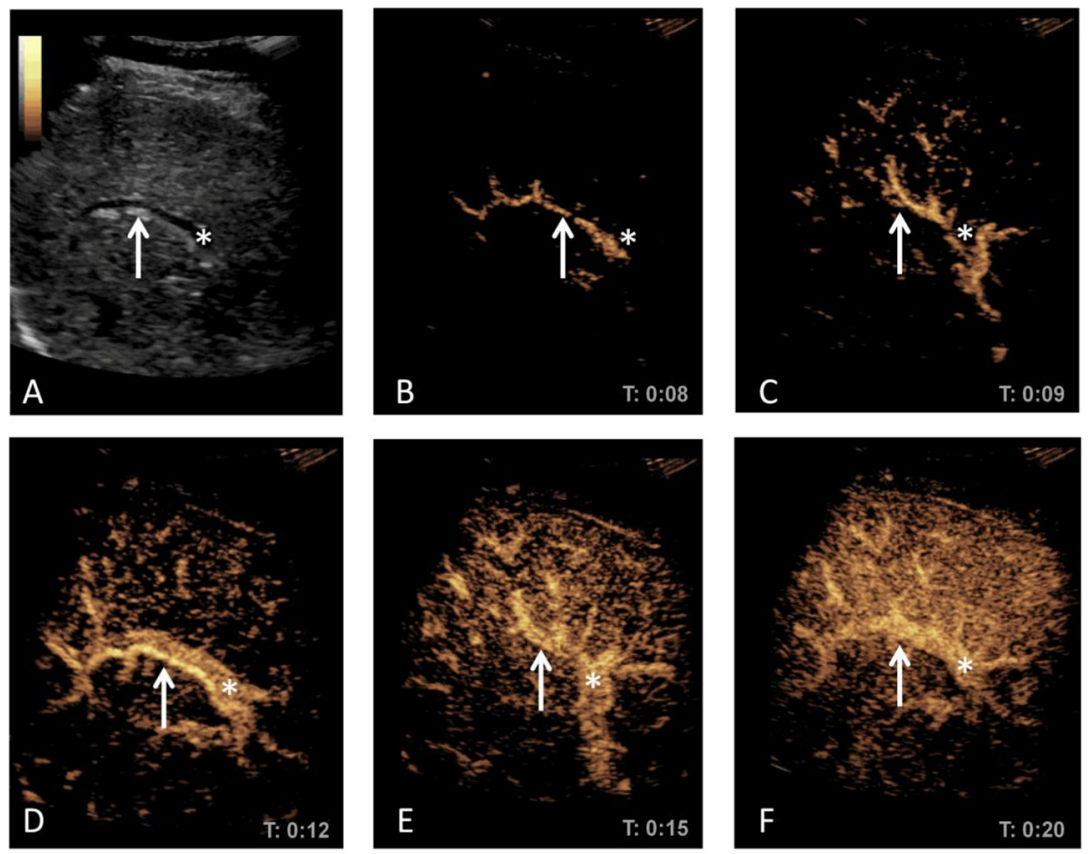

Figure 5.

Arterial phase of CEUS. B-mode ultrasound (A) and CEUS image (B) demonstrate first arrival of contrast agent into the right hepatic artery, which quickly progresses throughout the hepatic arterial circulation (C). Portal vein (asterisk) does not enhance at this stage. (D) arrival of contrast into the right portal vein (asterisk) which enhances less that hepatic artery (arrow). Progression of portal vein enhancement (E) which leads to uniform enhancement of hepatic parenchyma (F). Image reproduced with permission from the ACR. 


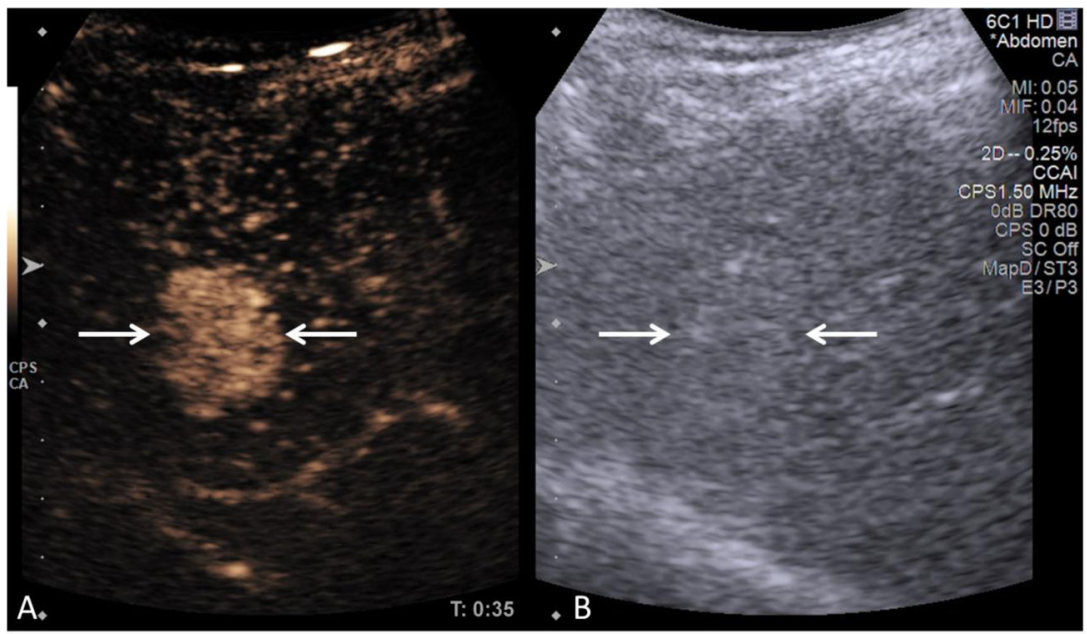

Figure 6.

Hyper-enhancement of the entire nodule in the arterial phase. Ill-defined focal liver nodule (arrows) on B-mode ultrasound (B) demonstrates uniform enhancement on contrastenhanced image (A) as compared to surrounding liver parenchyma at the same depth. Image reproduced with permission from the ACR. 


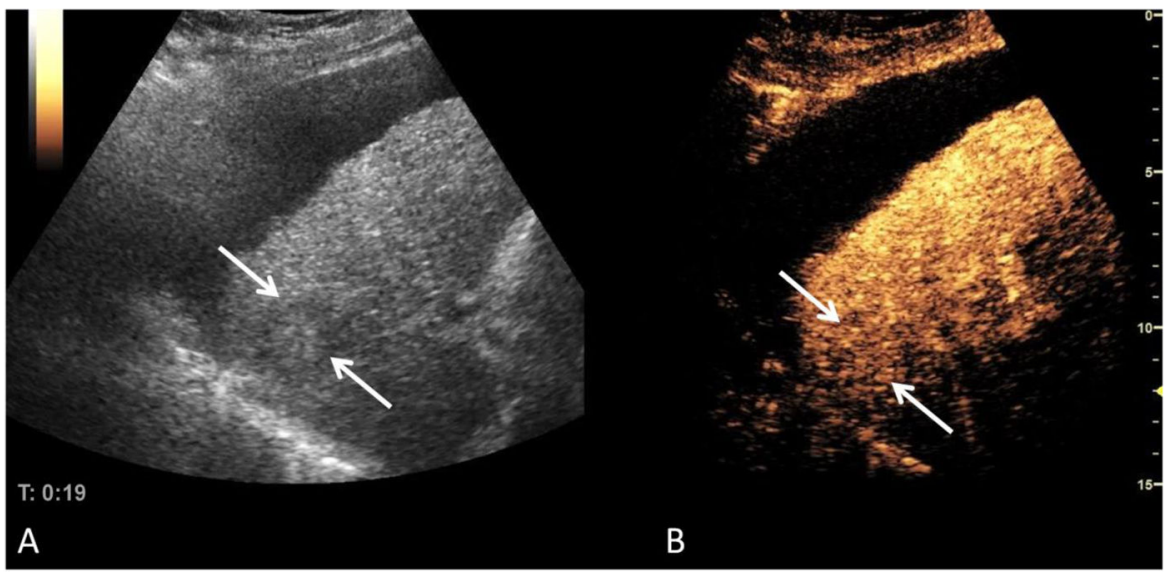

Figure 7.

Iso-enhancement of the entire nodule in the arterial phase. Indeterminate focal liver nodule (arrows) on B-mode ultrasound (A) demonstrates level of enhancement on contrastenhanced image (B) identical to surrounding liver parenchyma at the same depth. Image reproduced with permission from the ACR. 


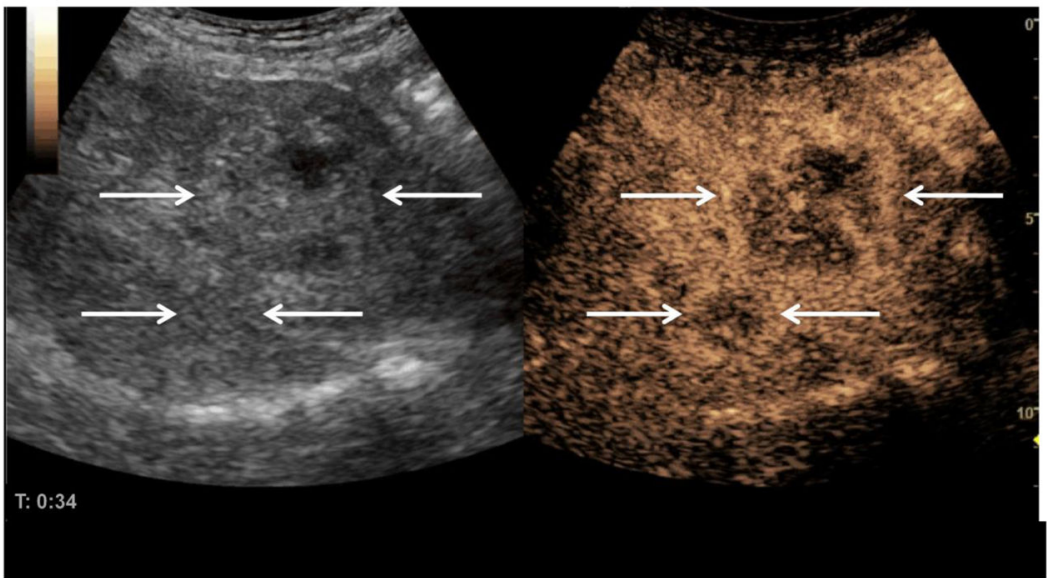

Figure 8.

Hypo-enhancement of the entire nodule in the arterial phase. Two heterogeneous focal liver nodules (arrows) barely visible on B-mode ultrasound (A) demonstrate significantly decreased arterial phase enhancement on contrast-enhanced image (B) as compared to surrounding liver parenchyma at the same depth. Image reproduced with permission from the ACR. 


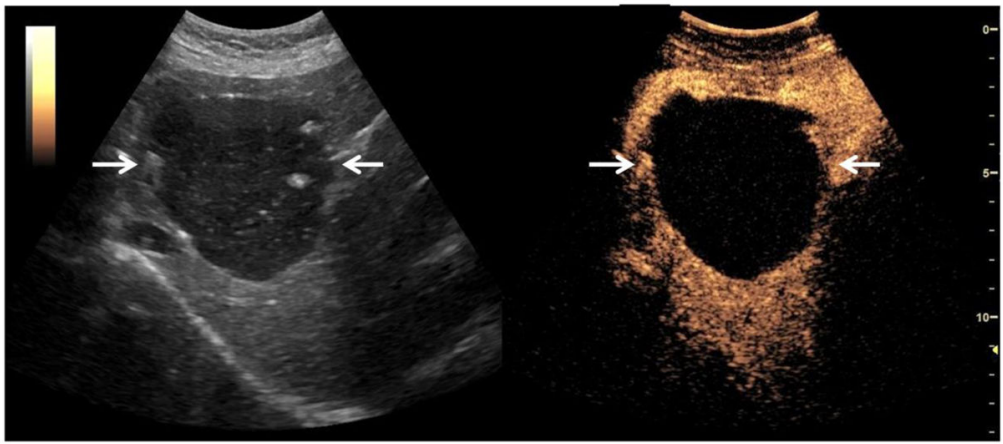

Figure 9.

No contrast enhancement. Heterogeneous liver nodule (arrows) on B-mode ultrasound (A) demonstrates no enhancement on contrast-enhanced image (B) as compared to surrounding liver parenchyma. Image reproduced with permission from the ACR. 


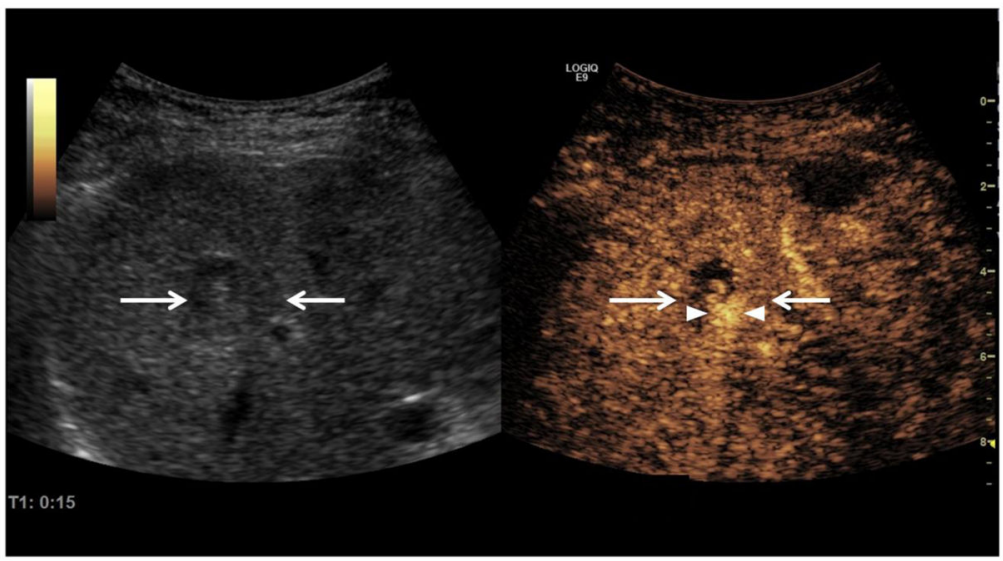

Figure 10.

Nodule-in-Nodule architecture. B-mode ultrasound (A) demonstrates slightly heterogeneous liver nodule (arrows). Contrast-enhanced image (B) demonstrates nodular area of hyperenhancement (arrowheads) within a larger nodule (arrows). Image reproduced with permission from the ACR. 


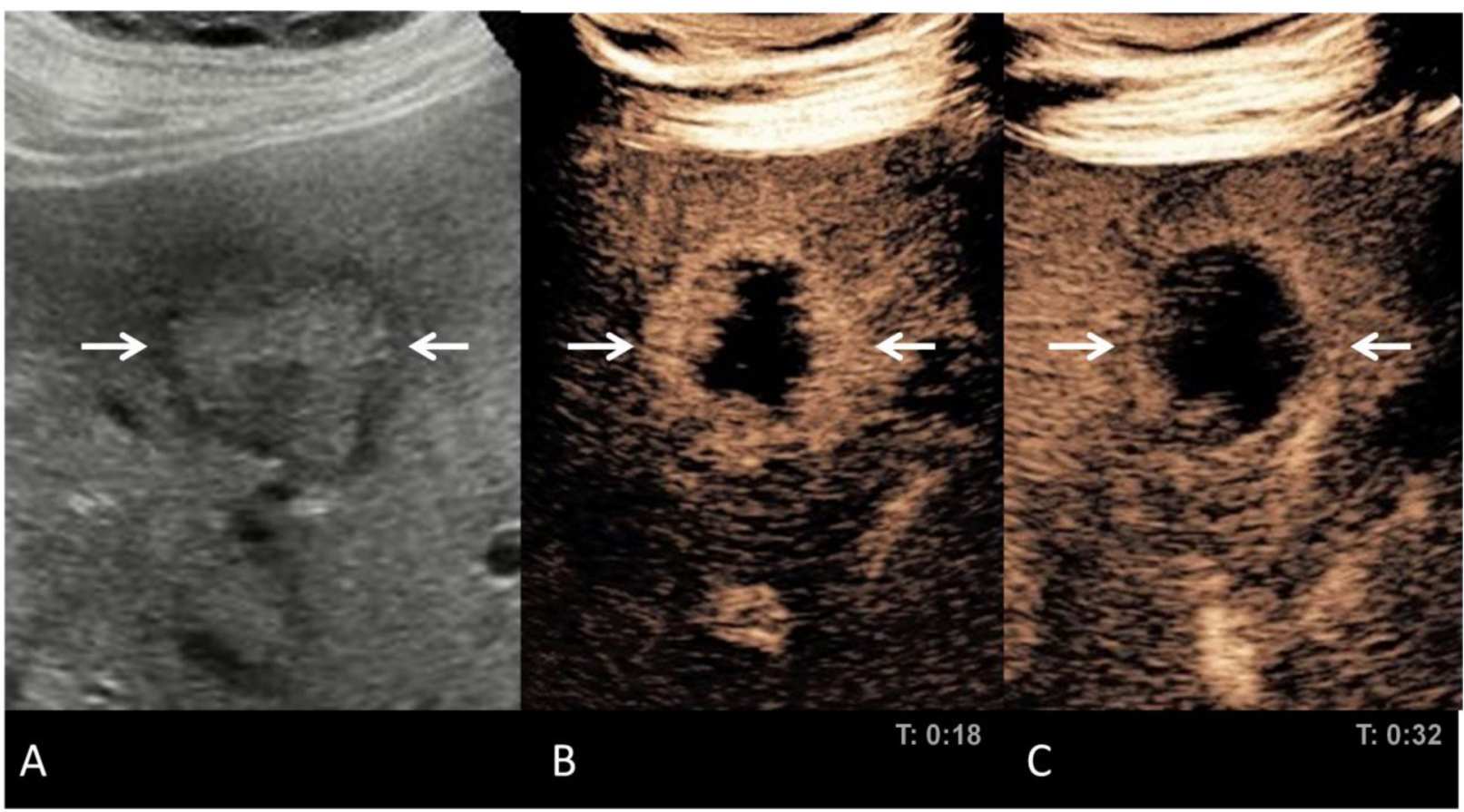

Figure 11.

Rim-enhancement of the focal liver nodule in the arterial phase. (A) B-mode image demonstrates heterogeneous nodule with hypoechoic halo (arrows). (B) Early arterial phase image shows irregular areas of increased enhancement along the periphery of the lesion, followed by rapid and marked washout (C). Image reproduced with permission from the ACR. 


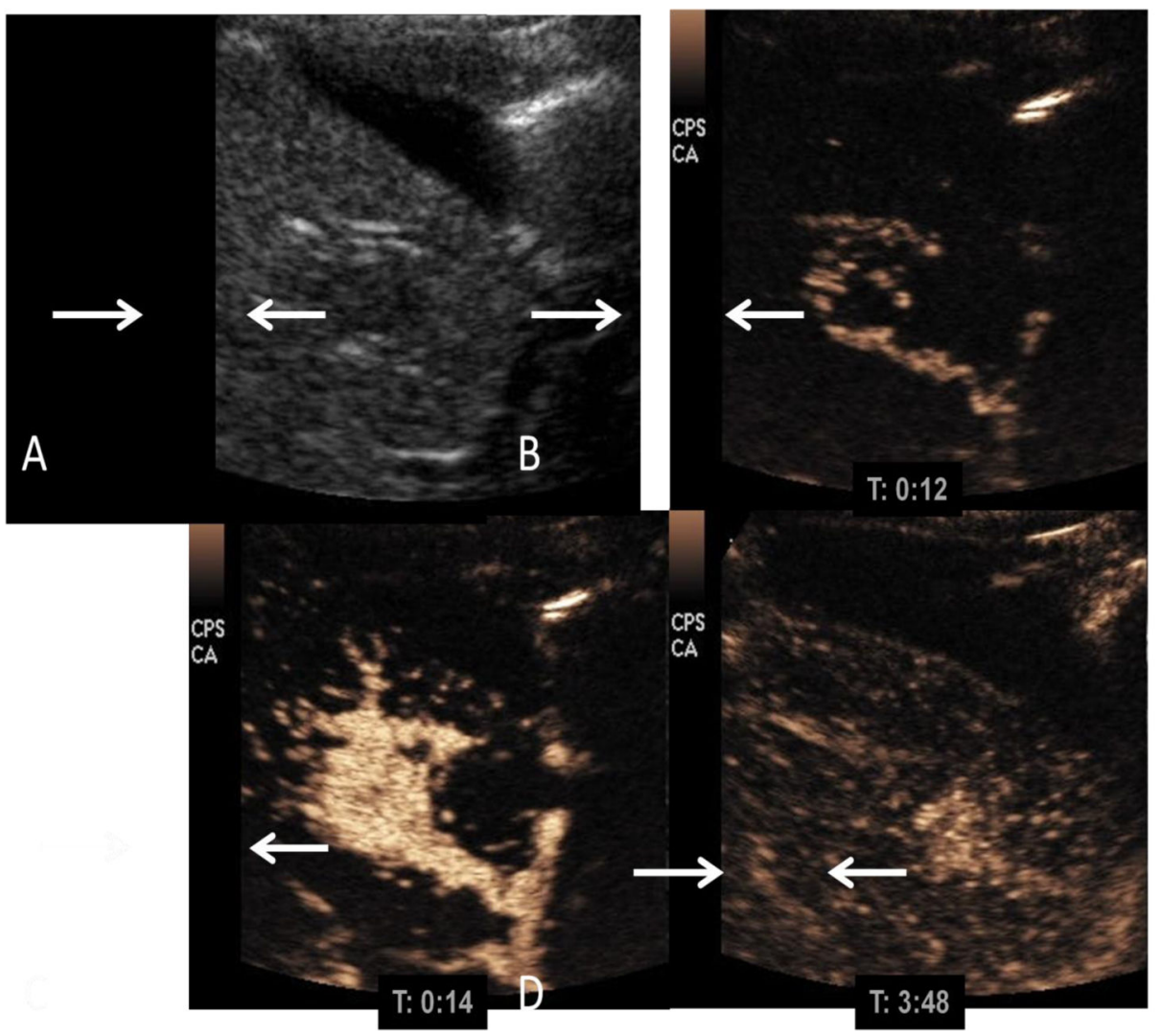

Figure 12.

Peripheral discontinuous globular enhancement. (A) B-mode image demonstrates small hypoechoic nodule (arrows). (B) Early arterial phase image shows small discontinuous globular areas of increased enhancement along the periphery of the lesion. This is followed by centripetal progression of the enhancement $(\mathrm{C})$ and sustained enhancement in the late phase (D). Image reproduced with permission from the ACR. 


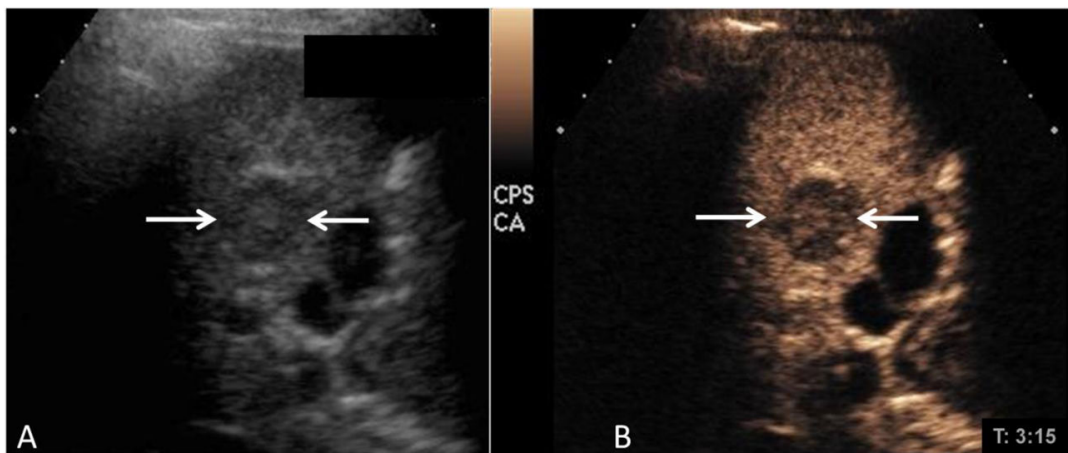

Figure 13.

Mild washout. Hypoechoic lesion (arrows) on B-mode ultrasound (A) becomes hypoechoic relative to the liver in the late phase but continues to show some contrast enhancement on contrast-enhanced image (B) at a time when the surrounding parenchyma is overtly enhanced. Image reproduced with permission from the ACR. 


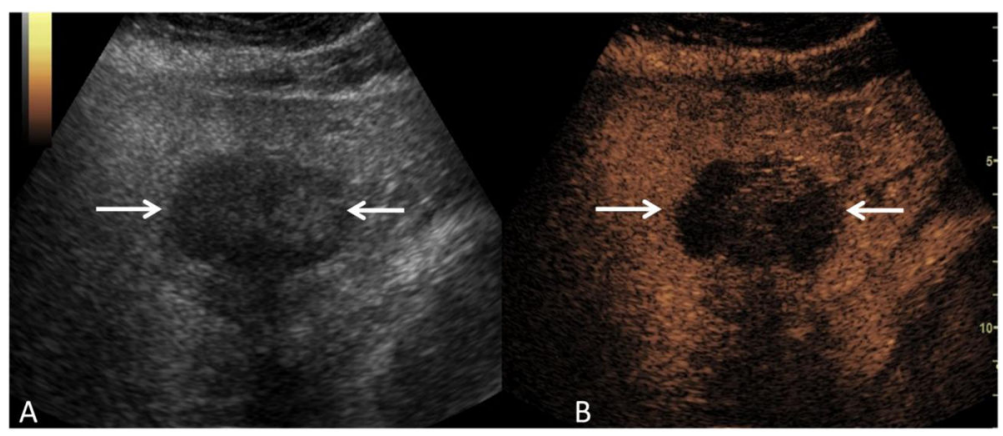

Figure 14.

Marked washout. Large hypoechoic lesion (arrows) on B-mode ultrasound (A) appears virtually devoid of contrast agent signal ("punched out") on contrast-enhanced image (B) at a time when the surrounding parenchyma is overtly enhanced. Image reproduced with permission from the ACR. 


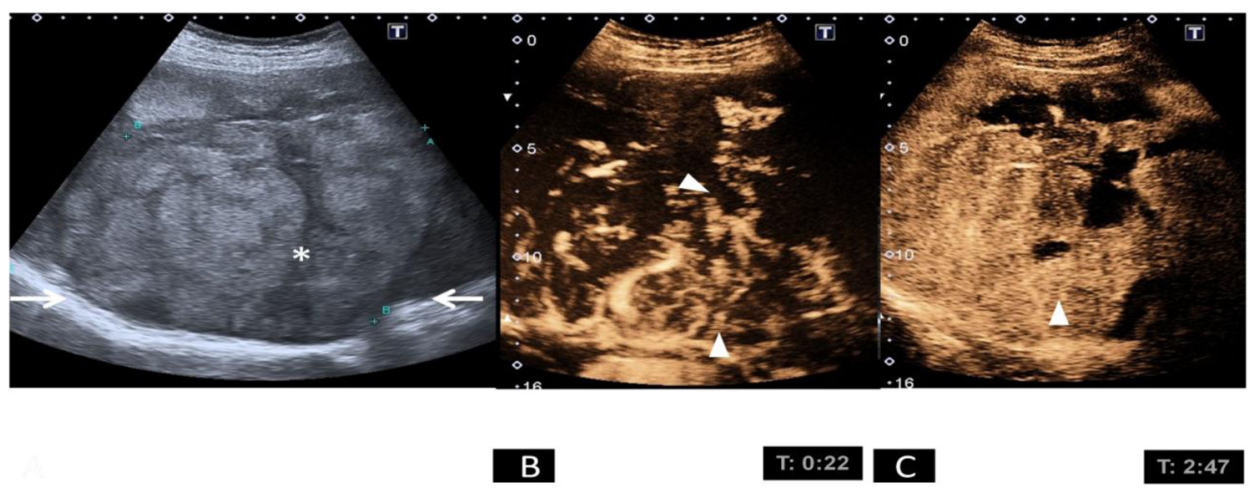

Figure 15.

Mosaic architecture. Large heterogeneous lesion (arrows) on B-mode ultrasound (A) demonstrates multiple confluent areas of tumor nodularity interspersed with fibrous septations (asterisk). On early arterial (B) and delayed phase (C) contrast-enhanced images tumor demonstrates heterogeneous enhancement with intervening areas hyper- and hypoenhancement (arrowheads). Image reproduced with permission from the ACR. 


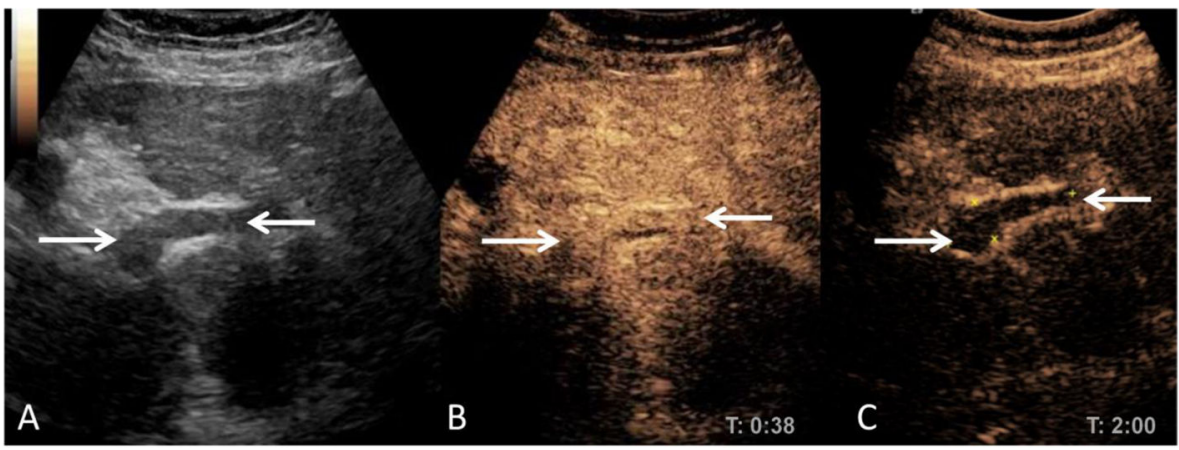

Figure 16.

Tumor in vein. B-mode ultrasound (A) demonstrates echogenic soft tissue in the lumen of portal vein (arrows). The soft tissue demonstrates arterial phase enhancement (B) and washout on delayed phase images (C). Image reproduced with permission from the ACR. 